\title{
Relations between regular A-optimal chemical and spring balance weighing designs with diagonal covariance matrix of errors
}

\author{
Bronisław Ceranka, Małgorzata Graczyk \\ Department of Mathematical and Statistical Methods, Poznań University of Life Sciences, \\ Wojska Polskiego 28, 60-637 Poznań, Poland, e-mail: bronicer@up.poznan.pl, \\ magra@up.poznan.pl
}

\section{SUMMARY}

\begin{abstract}
In this paper, we study the relationships between regular A-optimal spring balance weighing designs and regular A-optimal chemical balance weighing designs. We give the basic relation between these designs in the case where the errors are uncorrelated and they have different variances. We give some examples of methods of construction of such designs.
\end{abstract}

Key words: A-optimal design, chemical balance weighing design, spring balance weighing design

\section{Introduction}

The formulation of a standard weighing design problem requires some objects with unknown weights and a weight measuring device, popularly known as a balance. There are two types of balances in use. In a chemical balance, there are two pans and the objects can be placed in either pan. Then standard weights are used to attain balance. In a spring balance, any number of objects can be placed on the pan. Then the pointer provides a reading which represents the total weight of objects in the pan. Suppose specifically that there are $p$ objects of unknown true weights $w_{1}, w_{2}, \ldots, w_{p}$, and we wish to estimate them employing $n$ measuring operations using either a chemical balance or a spring 
balance. Let $y_{1}, y_{2}, \ldots, y_{n}$ denote respectively the observations recorded in these $n$ operations. It is assumed that the observations follow the linear model

$$
\mathbf{y}=\mathbf{X w}+\mathbf{e},
$$

where $\mathbf{y}=\left(y_{1}, y_{2}, \ldots, y_{n}\right)^{\prime}, \mathbf{w}=\left(w_{1}, w_{2}, \ldots, w_{p}\right)^{\prime}$, and $\mathbf{X}$ is a matrix of order $n \times p$ called the weighing design matrix. Its elements indicate in which combinations the objects are selected for the measurements. The elements of $\mathbf{X}$ are $x_{i j}, i=1,2, \ldots, n, j=1,2, \ldots, p$, and a typical element $x_{i j}$ is given by

$$
\begin{aligned}
x_{i j}=1 & \begin{array}{l}
\text { if the } j^{\text {th }} \text { object is placed on the right pan } \\
\text { during the } i^{\text {th }} \text { weighing operation, }
\end{array} \\
=-1 & \begin{array}{l}
\text { if the } j^{\text {th }} \text { object is placed on the left pan } \\
\text { during the } i \text { th }
\end{array} \\
=0 & \begin{array}{l}
\text { if the } j^{\text {th }} \text { object is not utilized in either pan } \\
\text { during the } i^{\text {th }} \text { weighing operation, }
\end{array}
\end{aligned}
$$

in the case of a chemical balance, and by

$$
\begin{aligned}
x_{i j}=1 & \begin{array}{l}
\text { if the } j^{\text {th }} \text { object is placed on the pan } \\
\text { during the } i^{\text {th }} \text { weighing operation, }
\end{array} \\
=0 & \begin{array}{l}
\text { if the } j^{\text {th }} \text { object is not utilized } \\
\text { during the } i^{\text {th }} \text { weighing operation, }
\end{array}
\end{aligned}
$$

in the case of a spring balance.

The vector $\mathbf{e}$ is the $n \times 1$ random vector of errors with $\mathrm{E}(\mathbf{e})=\mathbf{0}_{n}$, $\operatorname{Var}(\mathbf{e})=\sigma^{2} \mathbf{G}$, where $\mathbf{G}$ is the $n \times n$ diagonal positive definite matrix. The normal equations estimating $\mathbf{w}$ are

$$
\mathbf{X}^{\prime} \mathbf{G}^{-1} \mathbf{X} \hat{\mathbf{w}}=\mathbf{X G}^{-1} \mathbf{y}
$$

where $\hat{\mathbf{w}}$ is the column vector of the weights estimated by the least squares method. The parameter vector $\mathbf{w}$ is estimable if and only if $\operatorname{rank}(\mathbf{X})=p$, and in this case 


$$
\hat{\mathbf{w}}=\left(\mathbf{X}^{\prime} \mathbf{G}^{-1} \mathbf{X}\right)^{-1} \mathbf{X}^{\prime} \mathbf{G}^{-1} \mathbf{y}
$$

and the covariance matrix of $\hat{\mathbf{w}}$ is

$$
\operatorname{Var}(\hat{\mathbf{w}})=\sigma^{2}\left(\mathbf{X}^{\prime} \mathbf{G}^{-1} \mathbf{X}\right)^{-1}
$$

Some problems related to the weighing designs have appeared in the literature; see Raghavarao (1971), Banerjee (1975), Shah and Sinha (1989)

The optimality problem is concerned with efficient estimation in some sense by a proper choice of the design matrix $\mathbf{X}$ among many designs at our disposal. Considerations relating to determination of the optimal design appear in many publications, including Jacroux and Notz (1983), Kageyama and Saha (1983), Pukelsheim (1983), Abrego et al. (2003), Chadjiconstantinidis and Chadjipadelis (1994), and Neubauer and Pace (2010).

In many problems, the an A-optimal design is considered. For a given covariance matrix of errors $\sigma^{2} \mathbf{G}$, the A-optimal design is the design $\mathbf{X}$ for which the sum of variances of estimators of unknown parameters, i.e. $\operatorname{tr}\left(\mathbf{X}^{\prime} \mathbf{G}^{-1} \mathbf{X}\right)^{-1}$, is minimal. Moreover, a design for which the sum of variances of estimators for parameters attains the lowest bound is called a regular A-optimal design. The important point to note here is that in the set of design matrices $\mathbf{X}$, a regular A-optimal design may not exist, whereas an A-optimal design always exists. The concept of the A-optimality has been discussed in the literature. The best general references are Jacroux and Notz (1983), Ceranka and Katulska (2001), Ceranka and Graczyk (2004), Ceranka et al. (2006, 2007), Masaro and Wong (2008), and Graczyk (2011, 2012a,b).

For each structure of the covariance matrix of errors $\mathbf{G}$, we have to determine special conditions determining the optimal design and corresponding methods of construction. In the present paper we consider a special case of weighing designs: a design for which the matrix of errors is diagonal. In the remainder of the paper we shall consider $\mathbf{G}$ to be an $n \times n$ positive definite diagonal matrix. 
Ceranka and Katulska (2001) showed that for any nonsingular chemical balance weighing design $\mathbf{X}$ with the covariance matrix $\sigma^{2} \mathbf{G}$

$$
\operatorname{tr}\left(\mathbf{X}^{\prime} \mathbf{G}^{-1} \mathbf{X}\right)^{-1} \geq \frac{p}{\operatorname{tr}\left(\mathbf{G}^{-1}\right)}
$$

In the special case of a spring balance weighing design where bias is present, it can be assumed to be one object, and its value is estimated by taking the column of the elements 1 in $\mathbf{X}$ corresponding to the bias, i.e.

$$
\mathbf{X}=\left[\begin{array}{ll}
\mathbf{1}_{n} & \mathbf{X}_{1}
\end{array}\right]
$$

where $\mathbf{1}_{n}$ is the $n \times 1$ column vector of ones. Graczyk (2011) showed that for a biased spring balance weighing design $\mathbf{X}$ with the covariance matrix $\sigma^{2} \mathbf{G}$

$$
\operatorname{tr}\left(\mathbf{X}_{1}^{\prime} \mathbf{G}^{-1} \mathbf{X}_{1}\right)^{-1} \geq \frac{4(p-1)}{\operatorname{tr}\left(\mathbf{G}^{-1}\right)}
$$

\section{Regular A-optimal weighing designs}

Definition 2.1. Any nonsingular chemical balance weighing design $\mathbf{X}$ with the covariance matrix of errors $\sigma^{2} \mathbf{G}$ is said to be regular A-optimal if $\operatorname{tr}\left(\mathbf{X}^{\prime} \mathbf{G}^{-1} \mathbf{X}\right)^{-1}$ attains the lowest bound given by (5), i.e. if

$$
\operatorname{tr}\left(\mathbf{X}^{\prime} \mathbf{G}^{-1} \mathbf{X}\right)^{-1}=\frac{p}{\operatorname{tr}(\mathbf{G})^{-1}}
$$

Definition 2.2. Any nonsingular biased spring balance weighing design $\mathbf{X}$ with the covariance matrix of errors $\sigma^{2} \mathbf{G}$ is said to be regular A-optimal if $\operatorname{tr}\left(\mathbf{X}_{1}^{\prime} \mathbf{G}^{-1} \mathbf{X}_{1}\right)^{-1}$ attains the lowest bound given by (7), i.e. if

$$
\operatorname{tr}\left(\mathbf{X}_{1}^{\prime} \mathbf{G}^{-1} \mathbf{X}_{1}\right)^{-1}=\frac{4(p-1)}{\operatorname{tr}(\mathbf{G})^{-1}}
$$

The basic ideas and elementary properties of weighing designs are presented, for instance, in Jacroux and Notz (1983), Kageyama and Saha (1983), Ceranka and Katulska (2001) and Graczyk (2011). The following 
theorems may be summarized by saying that they present the necessary and sufficient conditions under which the chemical balance weighing design $\mathbf{X}$ with the covariance matrix $\sigma^{2} \mathbf{G}$, or the biased spring balance weighing design $\mathbf{X}$ with the same covariance matrix of errors $\sigma^{2} \mathbf{G}$, is a regular A-optimal design.

Theorem 2.1. For any positive definite $n \times n$ diagonal matrix $\mathbf{G}$, the $n \times p$ matrix $\mathbf{X}$ is the design matrix of a regular A-optimal chemical balance weighing design if and only if

$$
\mathbf{X}^{\prime} \mathbf{G}^{-1} \mathbf{X}=\operatorname{tr}(\mathbf{G})^{-1} \mathbf{I}_{p} .
$$

The above result was originally proved by Ceranka and Katulska in 2001.

For the case of a biased spring balance weighing design $\mathbf{X}$ we have the following result.

Theorem 2.2. Any nonsingular design matrix $\mathbf{X}$ of the form (6) with the diagonal covariance matrix of errors $\sigma^{2} \mathbf{G}$ is a regular A-optimal biased spring balance weighing design if and only if

$$
\mathbf{X}_{1}^{\prime} \mathbf{G}^{-1} \mathbf{X}_{1}=\frac{\operatorname{tr}(\mathbf{G})^{-1}}{4}\left(\mathbf{I}_{p-1}+\mathbf{1}_{p-1} \mathbf{1}_{p-1}^{\prime}\right)
$$

This theorem was proved by Graczyk (2011).

The aim of this paper is to bring together two areas of weighing designs chemical balance weighing designs and spring balance weighing designs - and to supplement the theory of weighing designs presented in the literature. We now consider the relationship between regular A-optimal chemical balance weighing designs and regular A-optimal biased spring balance weighing designs. The next theorem provides a characterization of these relations.

Theorem 2.3. A biased spring balance weighing design $\mathbf{X}$ of the form (6) with the covariance matrix $\sigma^{2} \mathbf{G}$ is a regular A-optimal design if and only if the chemical balance weighing design $\mathbf{X}^{*}=2 \mathbf{X}-\mathbf{1}_{n} \mathbf{1}_{p}^{\prime}$ with the same covariance matrix $\sigma^{2} \mathbf{G}$ is regular A-optimal. 
Proof. We begin by considering $\mathbf{X}$ in (6). $\mathbf{X}$ is the matrix of a regular A-optimal biased spring balance weighing design with the covariance matrix $\sigma^{2} \mathbf{G}$. Hence $\mathbf{X}^{*}=\left[\begin{array}{ll}\mathbf{1}_{n} & 2 \mathbf{X}_{1}-\mathbf{1}_{n} \mathbf{1}_{p-1}^{\prime}\end{array}\right]$ and we obtain

$$
\mathbf{X}^{* \prime} \mathbf{G}^{-1} \mathbf{X}^{*}=\left[\begin{array}{rr}
\mathbf{1}_{n}^{\prime} \mathbf{G}^{-1} \mathbf{1}_{n} & 2 \mathbf{1}_{n}^{\prime} \mathbf{G}^{-1} \mathbf{X}_{1}-\mathbf{1}_{n}^{\prime} \mathbf{G}^{-1} \mathbf{1}_{n} \mathbf{1}_{p-1}^{\prime} \\
2 \mathbf{X}_{1}^{\prime} \mathbf{G}^{-1} \mathbf{1}_{n}-\mathbf{1}_{p-1} \mathbf{1}_{n}^{\prime} \mathbf{G}^{-1} \mathbf{1}_{n} & \left(2 \mathbf{X}_{1}^{\prime}-\mathbf{1}_{p-1} \mathbf{1}_{n}^{\prime}\right) \mathbf{G}^{-1}\left(2 \mathbf{X}_{1}-\mathbf{1}_{n} \mathbf{1}_{p-1}^{\prime}\right)
\end{array}\right]
$$

From the above assumption it follows that condition (10) is satisfied. It is necessary to note that

$$
\mathbf{X}^{*^{\prime}} \mathbf{G}^{-1} \mathbf{X}^{*}=\left[\begin{array}{rr}
\mathbf{1}_{n}^{\prime} \mathbf{G}^{-1} \mathbf{1}_{n} & \mathbf{0}_{p-1}^{\prime} \\
\mathbf{0}_{p-1} & \left(\mathbf{1}_{n}^{\prime} \mathbf{G}^{-1} \mathbf{1}_{n}\right) \mathbf{I}_{p-1}
\end{array}\right]=\operatorname{tr}\left(\mathbf{G}^{-1}\right) \mathbf{I}_{p} .
$$

Consequently, $\mathbf{X}^{*}$ is the matrix of a regular A-optimal chemical balance weighing design with the covariance matrix $\sigma^{2} \mathbf{G}$.

Now, let $\mathbf{X}$ of the form (6) denote the matrix of a biased spring balance weighing design with the covariance matrix $\sigma^{2} \mathbf{G}$, while $\mathbf{X}^{*}=2 \mathbf{X}-\mathbf{1}_{n} \mathbf{1}_{p}^{\prime}$ is the matrix of a regular A-optimal chemical balance weighing design with the same covariance matrix $\sigma^{2} \mathbf{G}$. From (12) and (10), we conclude that

$$
2 \mathbf{1}_{n}^{\prime} \mathbf{G}^{-1} \mathbf{X}_{1}-\mathbf{1}_{n}^{\prime} \mathbf{G}^{-1} \mathbf{1}_{n} \mathbf{1}_{p-1}^{\prime}=\mathbf{0}_{p-1}^{\prime}
$$

and finally that

$$
4 \mathbf{X}_{1}^{\prime} \mathbf{G}^{-1} \mathbf{X}_{1}-2 \mathbf{1}_{p-1} \mathbf{1}_{n}^{\prime} \mathbf{G}^{-1} \mathbf{X}_{1}-2 \mathbf{X}_{1}^{\prime} \mathbf{G}^{-1} \mathbf{1}_{n} \mathbf{1}_{p-1}^{\prime}+\mathbf{1}_{p-1} \mathbf{1}_{n}^{\prime} \mathbf{G}^{-1} \mathbf{1}_{n} \mathbf{1}_{p-1}^{\prime}=\left(\mathbf{1}_{n}^{\prime} \mathbf{G}^{-1} \mathbf{1}_{n}\right) \mathbf{I}_{p-1}
$$

From this

$$
\mathbf{X}_{1}^{\prime} \mathbf{G}^{-1} \mathbf{X}_{1}=\frac{\operatorname{tr}(\mathbf{G})^{-1}}{4}\left(\mathbf{I}_{p-1}+\mathbf{1}_{p-1} \mathbf{1}_{p-1}^{\prime}\right)
$$

Furthermore, it follows that $\mathbf{X}$ of the form (6) is the matrix of a regular A-optimal biased spring balance weighing design with the covariance matrix $\sigma^{2} \mathbf{G}$. The theorem is therefore proved. 


\section{Examples}

Using the results obtained so far, the following examples of weighing designs can be established. Let us consider an experiment in which we estimate unknown measurements of $p=6$ objects in $n=11$ measurement operations. We work under the assumption that for the covariance matrix of errors $\sigma^{2} \mathbf{G}$, the matrix $\mathbf{G}$ is given by

$$
\mathbf{G}=\left[\begin{array}{ll}
0.5 & \mathbf{0}_{10}^{\prime} \\
\mathbf{0}_{10} & \mathbf{I}_{10}
\end{array}\right] \text {. }
$$

Thus we take the balanced incomplete block design with the parameters $v=p-1=5, b=n-1=10, r=4, k=2, \lambda=1$. Then the matrix of the biased spring balance weighing design can be expressed as

$$
\mathbf{X}^{\prime}=\left[\begin{array}{lllllllllll}
1 & 1 & 1 & 1 & 1 & 1 & 1 & 1 & 1 & 1 & 1 \\
1 & 1 & 1 & 1 & 1 & 0 & 0 & 0 & 0 & 0 & 0 \\
1 & 1 & 0 & 0 & 0 & 1 & 1 & 1 & 0 & 0 & 0 \\
1 & 0 & 1 & 0 & 0 & 1 & 0 & 0 & 1 & 1 & 0 \\
1 & 0 & 0 & 1 & 0 & 0 & 1 & 0 & 1 & 0 & 1 \\
1 & 0 & 0 & 0 & 1 & 0 & 0 & 1 & 0 & 1 & 1
\end{array}\right] .
$$

Applying (11) to the matrix $\mathbf{X}$, we obtain $\mathbf{X}_{1}^{\prime} \mathbf{G}^{-1} \mathbf{X}_{1}=3\left(\mathbf{I}_{5}+\mathbf{1}_{5} \mathbf{1}_{5}^{\prime}\right)$. Thus $\mathbf{X}$ is the design matrix of a regular A-optimal biased spring balance weighing design. Now, let $\mathbf{X}^{*}=2 \mathbf{X}-\mathbf{1}_{11} \mathbf{1}_{6}^{\prime}$ be the matrix of a chemical balance weighing design in the form

$$
\mathbf{X}^{*^{\prime}}=\left[\begin{array}{rrrrrrrrrrr}
1 & 1 & 1 & 1 & 1 & 1 & 1 & 1 & 1 & 1 & 1 \\
1 & 1 & 1 & 1 & 1 & -1 & -1 & -1 & -1 & -1 & -1 \\
1 & 1 & -1 & -1 & -1 & 1 & 1 & 1 & -1 & -1 & -1 \\
1 & -1 & 1 & -1 & -1 & 1 & -1 & -1 & 1 & 1 & -1 \\
1 & -1 & -1 & 1 & -1 & -1 & 1 & -1 & 1 & -1 & 1 \\
1 & -1 & -1 & -1 & 1 & -1 & -1 & 1 & -1 & 1 & 1
\end{array}\right] .
$$


For the matrix $\mathbf{X}^{*}$, one can easily prove that $\mathbf{X}^{*^{\prime}} \mathbf{G}^{-1} \mathbf{X}^{*}=12 \mathbf{I}_{6}$, because of (10). Then $\mathbf{X}^{*}$ is the design matrix of a regular A-optimal chemical balance weighing design.

For a deeper discussion of constructions of weighing designs, let us consider another example. In this experiment we estimate unknown measurements of $p=5$ objects in $n=11$ measurement operations. Our basic assumption is that for the covariance matrix of errors $\sigma^{2} \mathbf{G}$ the matrix $\mathbf{G}$ is given by

$$
\mathbf{G}=\left[\begin{array}{rrr}
2 \mathbf{I}_{2} & \mathbf{0}_{2} \mathbf{0}_{3}^{\prime} & \mathbf{0}_{2} \mathbf{0}_{6}^{\prime} \\
\mathbf{0}_{3} \mathbf{0}_{2}^{\prime} & 3 \mathbf{I}_{3} & \mathbf{0}_{3} \mathbf{0}_{6}^{\prime} \\
\mathbf{0}_{6} \mathbf{0}_{2}^{\prime} & \mathbf{0}_{6} \mathbf{0}_{3}^{\prime} & \mathbf{I}_{6}
\end{array}\right]
$$

In this case we choose the balanced incomplete block design with the parameters $v=p-1=4, b=n-5=6, r=3, k=2, \lambda=1$. Then the matrix of the biased spring balance weighing design can be described as follows:

$$
\mathbf{X}^{\prime}=\left[\begin{array}{lllllllllll}
1 & 1 & 1 & 1 & 1 & 1 & 1 & 1 & 1 & 1 & 1 \\
0 & 0 & 1 & 1 & 1 & 1 & 1 & 1 & 0 & 0 & 0 \\
0 & 0 & 1 & 1 & 1 & 1 & 0 & 0 & 1 & 1 & 0 \\
0 & 0 & 1 & 1 & 1 & 0 & 1 & 0 & 1 & 0 & 1 \\
0 & 0 & 1 & 1 & 1 & 0 & 0 & 1 & 0 & 1 & 1
\end{array}\right] .
$$

For the matrix $\mathbf{X}$, according to (11), $\mathbf{X}_{1}^{\prime} \mathbf{G}^{-1} \mathbf{X}_{1}=2\left(\mathbf{I}_{4}+\mathbf{1}_{4} \mathbf{1}_{4}^{\prime}\right)$. Then $\mathbf{X}$ is the matrix of a regular A-optimal biased spring balance weighing design. Now, let $\mathbf{X}^{*}=2 \mathbf{X}-\mathbf{1}_{11} \mathbf{1}_{5}^{\prime}$ be the design matrix of a chemical balance weighing design in the form

$$
\mathbf{X}^{*^{\prime}}=\left[\begin{array}{rrrrrrrrrrr}
1 & 1 & 1 & 1 & 1 & 1 & 1 & 1 & 1 & 1 & 1 \\
-1 & -1 & 1 & 1 & 1 & 1 & 1 & 1 & -1 & -1 & -1 \\
-1 & -1 & 1 & 1 & 1 & 1 & -1 & -1 & 1 & 1 & -1 \\
-1 & -1 & 1 & 1 & 1 & -1 & 1 & -1 & 1 & 1 & 1 \\
-1 & -1 & 1 & 1 & 1 & -1 & -1 & 1 & -1 & -1 & 1
\end{array}\right]
$$


For the matrix $\mathbf{X}^{*}$ we have $\mathbf{X}^{*} \mathbf{G}^{-1} \mathbf{X}^{*}=8 \mathbf{I}_{5}$, because of (10). From the above it follows that $\mathbf{X}^{*}$ is the design matrix of a regular A-optimal chemical balance weighing design.

\section{REFERENCES}

Abrego B., Fernandez-Merchant S., Neubauer G.N., Watkins W. (2003): D-optimal weighing designs for $n \equiv-1 \bmod 4$ objects and a large number of weighings. Linear Algebra and its Applications 374: 175-218.

Banerjee K.S. (1975): Weighing Designs for Chemistry, Medicine. Economics, Operations Research, Statistics. Marcel Dekker Inc., New York.

Ceranka B., Graczyk M. (2004): A-optimal chemical balance weighing design. Folia Facultatis Scientiarum Naturalium Universitatis Masarykianae Brunensis, Mathematica 15: 41-54.

Ceranka B., Graczyk M., Katulska K. (2006): A-optimal chemical balance weighing design with nonhomogeneity of variances of errors. Statistics and Probability Letters 76: 653 - 665

Ceranka B., Graczyk M., Katulska K. (2007): On certain A-optimal chemical balance weighing designs. Computational Statistics and Data Analysis 51: 5821-5827.

Ceranka B., Katulska K. (2001): A-optimal chemical balance weighing design with diagonal covariance matrix of errors. Moda 6, Advances in Model Oriented Design and Analysis, A.C. Atkinson, P. Hackl, W.G. Müller, eds., Physica-Verlag, Heidelberg, New York, 29-36.

Chadjiconstantinidis S., Chadjipadelis T. (1994): A construction method of new D-A-optimal designs when $N \equiv 3 \bmod 4$ and $k \leq N-1$. Discrete Mathematics 131: 39-50.

Graczyk M. (2011): A-optimal biased spring balance design. Kybernetika 47, 893-901.

Graczyk M. (2012a): Notes about A-optimal spring balance weighing design. Journal of Statistical Planning and Inference 142: 781-784.

Graczyk M. (2012b): Regular A-optimal spring balance weighing designs. Revstat 10: 323-333.

Jacroux M., Notz W. (1983): On the optimality of spring balance weighing designs. The Annals of Statistics 11: 970-978.

Kageyama S., Saha G.M. (1983): Note on the construction of optimum chemical balance weighing designs. Ann. Inst. Statist. Mat. 35A: 447-452.

Neubauer G.N., Pace R.G. (2010): D-optimal (0,1)-weighing designs for eight objects. Linear Algebra and its Applications 432: 2634-2657. 
Masaro J., Wong C.S. (2008): Robustness of A-optimal designs. Linear Algebra and its Applications 429: 1392-1408.

Pukelsheim F. (1993): Optimal Design of Experiment. John Wiley and Sons, New York.

Raghavarao D. (1971): Constructions and Combinatorial Problems in Designs of Experiments. John Wiley Inc., New York.

Shah K.R., Sinha B.K. (1989): Theory of Optimal Designs. Springer-Verlag, Berlin. 